\title{
Do Not Attempt Completion of Pointless Records - Or DNACPR
} Ted Willis ${ }^{*}$

Bridge Street Surgery, USA

*Corresponding author: Ted Willis, Bridge Street Surgery, USA, Tel: 01652 680789; E-mail: edmundawillis@gmail.com

Rec date: Jan 19, 2016, Acc date: Jan 20, 2016, Pub date: Jan 25, 2016

Copyright: (c) 2016 Wills T. This is an open-access article distributed under the terms of the Creative Commons Attribution License, which permits unrestricted use, distribution, and reproduction in any medium, provided the original author and source are credited.

\section{Introduction}

Many GPs are being asked to provide DNACPR forms for elderly patients in residential care. In our area, we have been told that the forms are invalid if they have been photocopied and do not have the original red border. We have also been told that we need to write new forms each time the ambulance service issues a new edition of the form.

We are told that without these forms, when patients die, staff will call 999 and that paramedics who find a patient who is not breathing will do CPR.

Of course, this would be distressing and undignified as well as a waste of ambulance resources. Dying elderly patients should of course be allowed to die naturally, and with dignity.

But is a DNACPR form necessary in order to achieve this?

I think it is not, because ambulance staff are professionals and know perfectly well that cardiac massage has no prospect of success in very elderly patients, so that even if care staff feels pressured into calling 999, paramedics should not initiate CPR in these patients.

Also the focus on DNACPR forms is a distraction from more important aspects of advance planning, such the decision as to whether the patient would wish to have antibiotic treatment for chest infections, or hospital admission, or intensive care. These are the important subjects for discussion with patients in their last years of life, but they are rarely addressed.

We should be talking to patients and their careers and we should include the subject of sudden collapse. Usually the plans would specify that 999 should not be called in the event of collapse. In order to protect the care home staff from the ire of the CQC etc., some sort of document will be needed, but it should not be limited to the form that the ambulance service produces. The present DNACPR form satisfies the needs of the ambulance service, but not ours, and certainly not the patients' needs.

Who should be taking the initiative to start these discussions? Ideally, a GP who knows the patient and knows the care staff. A good care home nurse would probably be nearly as good. If the patient lacks capacity we should ask relatives whether the patient has discussed the problem in the past. How should the consultation be recorded? In their medical records, of course with a copy for the care staff.

\section{Conclusion}

Many areas are making progress on this issue, but these initiatives need rolling out over the whole country. Meanwhile, we should refuse to provide the DNACPR forms, which are a pointless distraction. 